\title{
On a modification of some conditional Cauchy equation
}

\author{
MARCIN BALCEROWSKI
}

\begin{abstract}
We show connections between generalized versions of some conditional Cauchy equation and its basic form. As a consequence we obtain the solution of the generalized equations in some classes of regular functions.
\end{abstract}

Mathematics Subject Classification. 39B12, 39B52.

Keywords. Additive function, Conditional Cauchy equation, Semigroup.

The main subject of this paper is the functional equation

$$
f(x+f(y))=g\left(f^{k}(x)\right)+h\left(f^{l}(y)\right)
$$

and its special case

$$
f(x+f(y))=f^{k}(x)+f^{l}(y)
$$

where $k$ and $l$ are fixed natural numbers. In some particular cases Eq. (2) takes the form of well known equations. For instance, if $k=1$ and $l=2$, then Eq. (2) becomes the following conditional Cauchy equation

$$
f(x+f(y))=f(x)+f^{2}(y)
$$

and for $k=l=1$ Eq. (2) has the form

$$
f(x+f(y))=f(x)+f(y) .
$$

The general solution of Eq. (3), as well as (4), in the class of self-mappings of an abelian group was given by J. Dhombres in [2]. We present some properties of Eqs. (1) and (2); in particular, we show a connection between (1) and (3). This lets us solve Eqs. (1) and (2) in some classes of regular functions.

\section{General case}

In this section we work with Eq. (1). Theorem 1 below gives basic properties of this equation. 
Theorem 1. Let $(S,+)$ be an abelian cancellative semigroup with a zero and let $f, g, h: S \rightarrow S$. The triple $(f, g, h)$ satisfies equation (1) if, and only if there exist $x_{0}, y_{0} \in S$ such that for every $x, y \in S$ the following conditions hold:

$$
\begin{aligned}
f(x+f(y))+f^{2}(0) & =f(x+f(0))+f^{2}(y) \\
f(x+f(0)) & =g\left(f^{k}(x)\right)+x_{0} \\
f^{2}(x) & =h\left(f^{l}(x)\right)+y_{0} \\
x_{0}+y_{0} & =f^{2}(0) .
\end{aligned}
$$

Proof. Assume that the triple $(f, g, h)$ satisfies Eq. (1). Applying (1) with $y=0$ we get

$$
f(x+f(0))=g\left(f^{k}(x)\right)+h\left(f^{l}(0)\right)
$$

for every $x \in S$. In particular,

$$
f^{2}(0)=g\left(f^{k}(0)\right)+h\left(f^{l}(0)\right) .
$$

Using (1) with $x=0$ we obtain

$$
f^{2}(y)=g\left(f^{k}(0)\right)+h\left(f^{l}(y)\right)
$$

for every $y \in S$. Put $x_{0}=h\left(f^{l}(0)\right)$ and $y_{0}=g\left(f^{k}(0)\right)$. It follows from conditions (9)-(11) that equalities (6)-(8) are satisfied. Using already proved conditions and equality (1) we get

$$
f(x+f(0))+f^{2}(y)=g\left(f^{k}(x)\right)+x_{0}+y_{0}+h\left(f^{l}(y)\right)=f(x+f(y))+f^{2}(0)
$$

for every $x, y \in S$. This proves (5).

Conversely, assume that there exist $x_{0}, y_{0} \in S$ such that conditions (5)-(8) are satisfied for every $x, y \in S$. Then

$$
\begin{aligned}
f(x+f(y))+f^{2}(0) & =f(x+f(0))+f^{2}(y)=g\left(f^{k}(x)\right)+x_{0}+f^{2}(y) \\
& =g\left(f^{k}(x)\right)+x_{0}+y_{0}+h\left(f^{l}(y)\right) \\
& =g\left(f^{k}(x)\right)+h\left(f^{l}(y)\right)+f^{2}(0)
\end{aligned}
$$

for every $x, y \in S$. This in view of the cancellativity of $S$ means that the triple $(f, g, h)$ satisfies Eq. (1).

Corollary 1. Let $(G,+)$ be an abelian group and let $f, g, h: G \rightarrow G$. If the triple $(f, g, h)$ satisfies Eq. (1), then the function $-f+f(0)$ is a solution of equation (3).

Proof. Assume that the triple $(f, g, h)$ satisfies Eq. (1). Applying Theorem 1 we deduce that $f$ satisfies Eq. (5). Inserting $x-f(y)$ in place of $x$ in (5) we get

$$
f(x)+f^{2}(0)=f(x-f(y)+f(0))+f^{2}(y)
$$


for every $x, y \in G$. In particular,

$$
f(-f(y)+f(0))+f^{2}(y)=f(0)+f^{2}(0)
$$

for every $y \in G$. Using (12) and (13) we obtain

$f(x-f(y)+f(0))=f(x)+f^{2}(0)-f^{2}(y)=f(x)+f(-f(y)+f(0))-f(0)$

for every $x, y \in G$. Hence

$$
-f(x-f(y)+f(0))=-f(x)+f(0)-f(-f(y)+f(0))
$$

for every $x, y \in G$. Adding $f(0)$ to both sides of the last equality we see that the function $-f+f(0)$ satisfies Eq. (3). This completes the proof.

Now we consider regular solutions of Eq. (1).

Theorem 2. Let $(G,+)$ be an abelian topological group with the $T_{0}$ property and let $f, g, h: G \rightarrow G$. Assume that the group $<(f-f(0))(G)>$ is dense in $G$ and $f$ has a limit at a point. Then the triple $(f, g, h)$ satisfies Eq. (1) if, and only if there exist $x_{0}, z_{0} \in G$ and a continuous additive function $a: G \rightarrow G$ such that $f=a+z_{0}$ and for every $x \in G$ the following conditions hold

$$
\begin{aligned}
g\left(\left(a+z_{0}\right)^{k}(x)\right) & =a(x)+a\left(z_{0}\right)+z_{0}-x_{0} \\
h\left(\left(a+z_{0}\right)^{l}(x)\right) & =a^{2}(x)+x_{0} .
\end{aligned}
$$

Proof. Assume that the triple $(f, g, h)$ satisfies Eq. (1). By Corollary 1 the function $-f+f(0)$ is a solution of Eq. (3). Applying Theorem 9 and Lemma 4 from [1] we deduce that the function $-f+f(0)$ is additive and continuous. Putting $z_{0}=f(0)$ and $a=f-f(0)$ we see that $f=a+z_{0}$. It follows from Theorem 1 that there exist $x_{0}, y_{0} \in G$ such that conditions (5)-(8) are satisfied for every $x, y \in G$. In particular, $y_{0}=f^{2}(0)-x_{0}$. Using equalities (6) and (7) we deduce that conditions (14) and (15) hold for every $x \in G$.

Now assume that the right side of the implication in the Theorem is satisfied. The function $f$ is affine, and therefore equality (5) holds for every $y \in S$. Put $y_{0}=f^{2}(0)-x_{0}$. Then (8) holds and from (15) we obtain (7). Using (14) we get (6). This completes the proof.

From Theorem 2 we derive the following result.

Corollary 2. Let $f, g, h: \mathbb{R} \rightarrow \mathbb{R}$ be functions. Assume that the group $<(f-$ $f(0))(\mathbb{R})>$ is dense in $\mathbb{R}$ and $f$ has a finite limit at a point. Then the triple $(f, g, h)$ satisfies Eq. (1) if, and only if there exists an $a \in \mathbb{R} \backslash\{0\}$ and $x_{0}, z_{0} \in \mathbb{R}$ such that $f(x)=a x+z_{0}$ and the following conditions hold:

$$
g(x)=a^{1-k}\left(x-z_{0} \sum_{i=0}^{k-1} a^{i}\right)+a z_{0}+z_{0}-x_{0}
$$


and

$$
h(x)=a^{2-l}\left(x-z_{0} \sum_{i=0}^{l-1} a^{i}\right)+x_{0}
$$

for every $x \in \mathbb{R}$.

Proof. Assume that the triple $(f, g, h)$ satisfies Eq. (1). By Theorem 2 there exist $x_{0}, z_{0} \in \mathbb{R}$ and an $a \in \mathbb{R}$ such that the equality $f(x)=a x+z_{0}$ and conditions (14) and (15) are satisfied for every $x \in \mathbb{R}$. Since the set $<(f-$ $f(0))(\mathbb{R})>$ is dense in $\mathbb{R}$, we have $a \neq 0$. By induction it is easy to prove that $f^{n}(x)=a^{n} x+z_{0} \sum_{i=0}^{n-1} a^{i}$ for every $x \in \mathbb{R}$ and $n \in \mathbb{N}$. Using (14) and (15) we obtain

$$
g\left(a^{k} x+z_{0} \sum_{i=0}^{k-1} a^{i}\right)=a x+a z_{0}+z_{0}-x_{0}
$$

and

$$
h\left(a^{l} x+z_{0} \sum_{i=0}^{l-1} a^{i}\right)=a^{2} x+x_{0}
$$

for every $x \in \mathbb{R}$. Hence $g\left(x+z_{0} \sum_{i=0}^{k-1} a^{i}\right)=a^{1-k} x+a z_{0}+z_{0}-x_{0}$ and $h(x+$ $\left.z_{0} \sum_{i=0}^{l-1} a^{i}\right)=a^{2-l} x+x_{0}$ for every $x \in \mathbb{R}$. Inserting here $x-z_{0} \sum_{i=0}^{k-1} a_{i}$ and, respectively, $x-z_{0} \sum_{i=0}^{l-1} a_{i}$ we see that conditions (16) and (17) hold for every $x \in \mathbb{R}$. This completes the proof.

\section{Case $g=h=\mathrm{id}$}

Now we consider Eq. (2). We start with some auxiliary results which present general properties of this equation.

Lemma 1. Let $(G,+)$ be a set with a binary operation and let $f: G \rightarrow G$ be a solution of Eq. (2). Then for every $n \in \mathbb{N}$ and $x, y \in G$ the following condition holds:

$$
f^{n}(x+f(y))=f^{k n}(x)+f^{(l-1) n+1}(y) .
$$

Moreover, for every $n \in \mathbb{N}$ the function $f^{n}$ satisfies $E q$. (2).

Proof. It follows from (2) that if $n=1$, then condition (20) is satisfied for every $x, y \in G$. Fix an $n \in \mathbb{N}$ and assume that (20) is satisfied for every $x, y \in G$. Then for every $x, y \in G$ we have

$$
\begin{aligned}
f^{n+1}(x+f(y)) & =f\left(f^{n}(x+f(y))=f\left(f^{k n}(x)+f^{(l-1) n+1}(y)\right)\right. \\
& =f\left(f^{k n}(x)+f\left(f^{(l-1) n}(y)\right)\right)=f^{k}\left(f^{k n}(x)\right)+f^{l}\left(f^{n l-n}(y)\right) \\
& =f^{k(n+1)}(x)+f^{(l-1)(n+1)+1}(y) .
\end{aligned}
$$

This finishes the inductive proof of (20). 
For the proof of the second part fix any $n \in \mathbb{N}$. Inserting $f^{n-1}(y)$ in place of $y$ in (20) we get

$$
f^{n}\left(x+f^{n}(y)\right)=f^{k n}(x)+f^{l n}(y)
$$

for every $x, y \in G$. Therefore $f^{n}$ satisfies Eq. (2).

Lemma 2. Let $(S,+)$ be an abelian cancellative semigroup and let $f: S \rightarrow S$ be a solution of Eq. (2). Then

$$
f^{k^{2}}(x)+f^{2 l-1}(y)=f^{k}(x)+f^{l^{2}-l+1}(y)
$$

for every $x, y \in S$. In particular, if $l \in\{1,2\}$, then

$$
f^{k^{2}}=f^{k}
$$

and $f$ satisfies the equation

$$
f^{k}\left(x+f^{k}(y)\right)=f^{k}(x)+f^{l k}(y) .
$$

Proof. Using Lemma 1 we deduce that $f$ satisfies condition (20) for every $x, y \in S$ and for every $n \in \mathbb{N}$. Hence

$$
f(x+f(y)+f(z))=f^{k}(x+f(y))+f^{l}(z)=f^{k^{2}}(x)+f^{l k-k+1}(y)+f^{l}(z)
$$

for every $x, y, z \in S$. Inserting here $f^{k-1}(y)$ in place of $y$ and $f^{l-1}(z)$ in place of $z$ we obtain

$$
f\left(x+f^{k}(y)+f^{l}(z)\right)=f^{k^{2}}(x)+f^{k l}(y)+f^{2 l-1}(z)
$$

for every $x, y, z \in S$. On the other hand, making use of (2) and 20 we get

$$
\begin{aligned}
f\left(x+f^{k}(y)+f^{l}(z)\right) & =f\left(x+f(y+f(z))=f^{k}(x)+f^{l}(y+f(z))\right. \\
& =f^{k}(x)+f^{k l}(y)+f^{l^{2}-l+1}(z)
\end{aligned}
$$

for every $x, y, z \in S$. The semigroup $S$ is abelian and cancellative and therefore, comparing the right sides of equalities (24) and (25), we obtain the first statement of the Lemma.

Now assume that $l \in\{1,2\}$. Then $l^{2}-l+1=2 l-1$ and, using (21) and the commutativity and cancellativity of $S$ again, we get (22). By virtue of Lemma 1 the function $f^{k}$ satisfies Eq. (2). This in view of (22) means that $f$ satisfies Eq. (23).

Lemma 3. Let $(S,+)$ be an abelian cancellative semigroup with a zero. If $f: S \rightarrow S$ satisfies Eq. (2), then

$$
f^{k+l-2}(0)=0 .
$$


Proof. We may assume that $\max \{k, l\} \geq 2$. First we consider the case $k \geq 2$. Inserting $f^{k-2}(y)$ in place of $y$ in (2) we obtain the condition

$$
f\left(x+f^{k-1}(y)\right)=f^{k}(x)+f^{k+l-2}(y)
$$

for every $x, y \in S$. Applying them for $x=y=0$ we get $f^{k}(0)=f^{k}(0)+$ $f^{k+l-2}(0)$, which, by virtue of the cancellativity of $S$, implies $f^{k+l-2}(0)=0$.

Now assume that $k=1$. Then $l \geq 2$. It follows from Lemma 2 that $f$ satisfies Eq. (21). Hence

$$
f^{l^{2}-l+1}=f^{2 l-1} .
$$

It follows from Lemma 1 that for every $n \in \mathbb{N}$ and for every $x, y \in S$ condition (20) is satisfied. Applying them for $n=l-1$ we get

$$
f^{l-1}(x+f(y))=f^{l-1}(x)+f^{l^{2}-2 l+2}(y)
$$

for every $x, y \in S$. Inserting $f^{l-2}(y)$ in place of $y$ in (28) we obtain

$$
f^{l-1}\left(x+f^{l-1}(y)\right)=f^{l-1}(x)+f^{l^{2}-l}(y)
$$

for every $x, y \in S$. Condition (29) with $f^{l-1}(x)$ in place of $x$ gives

$$
f^{l-1}\left(f^{l-1}(x)+f^{l-1}(y)\right)=f^{2 l-2}(x)+f^{l^{2}-l}(y)
$$

for every $x, y \in S$. The left-hand side of the last equality is symmetric with respect to replacing $x$ and $y$ and thus its right-hand side also has this property. Therefore

$$
f^{2 l-2}(x)+f^{l^{2}-l}(y)=f^{2 l-2}(y)+f^{l^{2}-l}(x)
$$

for every $x, y \in S$. Inserting $f(y)$ in place of $y$ in the last condition we get

$$
f^{2 l-2}(x)+f^{l^{2}-l+1}(y)=f^{2 l-1}(y)+f^{l^{2}-l}(x)
$$

for every $x, y \in S$. Since $S$ is abelian and cancellative, using (27) and (30) we obtain $f^{l^{2}-l}(x)=f^{2 l-2}(x)$ for every $x \in S$. Hence, in view of equality (29), we deduce that $f^{l-1}$ satisfies Eq. (3). In particular, $f^{2 l-2}(0)=f^{l-1}\left(0+f^{l-1}(0)\right)=$ $f^{l-1}(0)+f^{2 l-2}(0)$, which in view of the cancellativity of $S$ gives $f^{l-1}(0)=0$. Therefore condition (26) follows with $k=1$. This completes the proof.

Lemma 4. Let $(S,+)$ be an abelian cancellative semigroup with a zero. Assume that

$$
(k+l-2) x \neq 0 \quad \text { for every } x \in S \backslash\{0\} .
$$

If $f: S \rightarrow S$ is a solution of $E q$. (2), then $f(0)=0$.

Proof. Assume that $f: S \rightarrow S$ is a solution of Eq. (2). We may assume that $S \neq\{0\}$. It follows from Lemma 3 that $f$ satisfies condition (26). In particular,

$$
f^{k+l-1}(0)=f(0) \text {. }
$$

We will show that

$$
f^{k+n}(0)=f^{k}(0)+f^{n}(0)
$$


for every $n \in \mathbb{N} \cup\{0\}$. If $n=0$, then the equality above is obvious. Fix an $n \in \mathbb{N} \cup\{0\}$ and assume that equality (33) is satisfied. Using equalities (2) and (32) we obtain

$$
\begin{aligned}
f^{k+n+1}(0) & =f\left(f^{k+n}(0)\right)=f^{k}(0)+f^{l+k+n-1}(0)=f^{k}(0)+f^{n}(f(0)) \\
& =f^{k}(0)+f^{n+1}(0) .
\end{aligned}
$$

This finishes the inductive proof.

Applying condition (33) with $n=l-1$ and making use of (32) we get

$$
f^{k}(0)+f^{l-1}(0)=f(0) .
$$

Now we show that

$$
f^{n}(0)=n f(0)
$$

for every $n \in \mathbb{N} \cup\{0\}$. For $n=0$ equality (35) holds. Fix an $n \in \mathbb{N} \cup\{0\}$ and assume that (35) is satisfied. Since $S \neq\{0\}$, it follows from (31) that $\max \{k, l\} \geq 2$. Therefore $k+l \geq 3$ and using equalities (26), (2), (33) and (34) we obtain

$$
\begin{aligned}
f^{n+1}(0) & =f\left(f^{n}(0)\right)=f\left(f^{n}(0)+f^{l+k-2}(0)\right)=f\left(f^{n}(0)+f\left(f^{l+k-3}(0)\right)\right) \\
& =f^{k+n}(0)+f^{2 l+k-3}(0)=f^{k}(0)+f^{n}(0)+f^{l-1}\left(f^{l+k-2}(0)\right) \\
& =f^{k}(0)+f^{n}(0)+f^{l-1}(0)=f^{n}(0)+f(0)=n f(0)+f(0) \\
& =(n+1) f(0) .
\end{aligned}
$$

This finishes the inductive proof of (35). Making use of (35) with $n=k+l-2$ and applying $(26)$ we get $(k+l-2) f(0)=0$. Using $(31)$ we obtain the assertion.

Making use of Theorem 1 and Lemma 4 we will prove the following result.

Theorem 3. Let $(S,+)$ be an abelian cancellative semigroup with a zero. Assume that $S$ satisfies condition (31). Let $f: S \rightarrow S$ be a function. Then $f$ satisfies Eq. (2) if, and only if it satisfies Eq. (3) and the condition

$$
f^{k}=f \text { and } f^{l}=f^{2} .
$$

Proof. Assume that $f$ satisfies Eq. (2). Applying Theorem 1 with $g=h=$ id we deduce that there exist $x_{0}, y_{0} \in S$ such that $f$ satisfies conditions (5)-(8) for every $x, y \in S$. It follows from Lemma 4 that $f(0)=0$. Therefore, making use of (5), we see that $f$ satisfies Eq. (3). Using (6) with $x=0$ we obtain $x_{0}=0$. Hence, by virtue of $(8)$, we get $y_{0}=0$. Making use of equalities (6) and $(7)$ we deduce that condition (36) is satisfied. The converse is obvious.

From Theorem 3 we derive results concerning regular solutions of Eq. (2). 
Theorem 4. Let $(G,+)$ be an abelian topological group [real linear topological space] with the $T_{0}$ property. Assume that $G$ satisfies condition (31). Let $f: G \rightarrow$ $G$ be a function such that $f$ has a limit at a point and the group $\langle f(G)\rangle$ is dense in $G$. Then $f$ satisfies Eq. (2) if, and only if $f$ is a continuous additive [linear] mapping satisfying the condition

$$
f^{k-1}=\mathrm{id} \text { and } f^{|l-2|}=\mathrm{id} .
$$

Proof. Assume that $f$ satisfies Eq. (2). It follows from Lemma 4 that $f(0)=0$. Applying Theorem 2 with $g=h=$ id we deduce that $f$ is a continuous affine function. Therefore $f$ is additive. In particular, the set $f(G)$ is a subgroup of $G$. Hence in view of the assumption we infer that $f(G)$ is dense in $G$. By Theorem 3 the function $f$ satisfies equalities (36). This means that $f^{k-1}$ and $f^{|l-2|}$ are equal to the identity function on $f(G)$. Making use of the density of $f(G)$ in $G$ and the continuity of $f$ we deduce that $f$ satisfies condition (37). The converse follows from Theorem 3 .

Corollary 3. Let $\max \{k, l\} \geq 2$ and let $f: \mathbb{R} \rightarrow \mathbb{R}$ be a function. Assume that $f$ has a finite limit at a point and the group $\langle f(\mathbb{R})>$ is dense in $\mathbb{R}$. Then $f$ satisfies Eq. (2) if, and only if one of the following conditions holds:

$\left(p_{1}\right) k=1, l=2$ and there exists an $a \in \mathbb{R} \backslash\{0\}$ such that $f(x)=$ ax for every $x \in \mathbb{R}$;

$\left(p_{2}\right) k$ is odd and $l$ is even, $k \neq 1$ or $l \neq 2$, and there exists an $a \in\{-1,1\}$ such that $f(x)=$ ax for every $x \in \mathbb{R}$;

$\left(p_{3}\right) k$ is even or $l$ is odd, and $f=\mathrm{id}$.

Proof. It is easy to check that the functions presented in cases $\left(p_{1}\right)-\left(p_{3}\right)$ are solutions of Eq. (2). So assume that $f$ satisfies Eq. (2). By Theorem 4 the function $f$ is a linear mapping and it satisfies equalities (37). Therefore there exists an $a \in \mathbb{R}$ such that

$$
f(x)=a x
$$

for every $x \in \mathbb{R}$. Since $\langle f(\mathbb{R})>$ is dense in $\mathbb{R}$, we have $a \neq 0$.

If $k=1$ and $l=2$, then, using (38), we complete the proof. Assume that $k \neq 1$ or $l \neq 2$. It follows from (37) that

$$
a^{k-1}=1 \quad \text { and } \quad a^{|l-2|}=1 .
$$

If $k$ is even or $l$ is odd, then condition (39) yields $a=1$, and thus $f=$ id. If $k$ is odd and $l$ is even, then (39) implies that $a \in\{-1,1\}$ and therefore either $f=-\mathrm{id}$, or $f=\mathrm{id}$. This completes the proof.

Proposition 3.2 [2] presents the general solution of Eq. (3) in the class of continuous functions mapping $\mathbb{R}$ into itself. In the next Corollary we extend that result to Eq. (2). 
Corollary 4. Let $\max \{k, l\} \geq 2$ and let $f: \mathbb{R} \rightarrow \mathbb{R}$ be a continuous function. The function $f$ satisfies Eq. (2) if, and only if one of the following conditions holds:

$\left(w_{1}\right) k=1, l=2$ and there exists an $a \in \mathbb{R}$ such that $f(x)=$ ax for every $x \in \mathbb{R}$;

$\left(w_{2}\right) k$ is odd and $l$ is even, $k \neq 1$ or $l \neq 2$, and there exists an $a \in\{-1,0,1\}$ such that $f(x)=$ ax for every $x \in \mathbb{R}$;

$\left(w_{3}\right) k$ is even or $l$ is odd, and there exists an $a \in\{0,1\}$ such that $f(x)=a x$ for every $x \in \mathbb{R}$.

Proof. Assume that $f$ satisfies Eq. (2). If $f$ is constant, then it follows from (2) that $f=0$. Otherwise the function $f$ is non-constant and continuous and therefore the set $f(\mathbb{R})$ is an interval with non-empty interior. Hence $\langle f(\mathbb{R})\rangle=\mathbb{R}$ and making use of Corollary 3 we get the suitable form of $f$. To prove the converse it is enough to apply Corollary 3 and to observe that the zero function satisfies Eq. (2).

Open Access. This article is distributed under the terms of the Creative Commons Attribution 4.0 International License (http://creativecommons.org/licenses/by/4.0/), which permits unrestricted use, distribution, and reproduction in any medium, provided you give appropriate credit to the original author(s) and the source, provide a link to the Creative Commons license, and indicate if changes were made.

\section{References}

[1] Balcerowski, M.: On the functional equation $f(x+g(y))-f(y+g(y))=f(x)-f(y)$ on groups. Aequationes Math. 78, 247-255 (2009)

[2] Dhombres, J.: Some aspects of functional equations. Lecture Notes. Chulalongkorn University, Department of Mathematics, Bangkok (1979)

Marcin Balcerowski

ul. 1 Maja 56/80

41-200 Sosnowiec

Poland

e-mail: mbalcerowski@math.us.edu.pl

Received: November 30, 2015

Revised: March 6, 2017 